\title{
Method development and initial results of testing for perfluorooctane sulfonate (PFOS) and perfluorooctanoic acid (PFOA) in waterproof sunscreens
}

\author{
Sasipin Keawmanee ${ }^{1}$, Suwanna Kitpati Boontanon ${ }^{{ }^{\dagger}}$, Narin Boontanon ${ }^{2}$ \\ ${ }^{1}$ Department of Civil and Environmental Engineering, Faculty of Engineering, Mahidol University, Nakhon Pathom 73170, Thailand \\ ${ }^{2}$ Faculty of Environment and Resource Studies, Mahidol University, Nakhon Pathom 73170, Thailand
}

\begin{abstract}
Perfluorooctane sulfonate (PFOS) and Perfluorooctanoic acid (PFOA) are persistent environmental pollutants, extremely stable, and possibly adversely affect human health. They are widely used in many industries and consumer goods, including sunscreen products. These substances are stable chemicals made of long carbon chains, having both lipid- and water-repellent qualities. The research objectives are (1) to find the most effective method for the preparation of semi-liquid samples by comparing solid phase extraction (SPE) and centrifugation after Pressurized liquid extraction (PLE), and (2) to determine the contamination levels of PFOS and PFOA in waterproof sunscreen samples. All sunscreen samples were analyzed by liquid chromatography coupled with tandem mass spectrometry (LC-MS/MS). Sunscreen samples were purchased from domestic and international brands sold in Thailand. Special chemical properties were considered for the selection of samples, e.g., those found in waterproof, sweat resistant, water resistant, and non-stick products. Considering the factors of physical properties, e.g., operation time, chemical consumption, and recovery percentage for selecting methods to develop, the centrifugation method using $2 \mathrm{~mL}$ of extracted sample with the conditions of $12,000 \mathrm{rpm}$ and $5^{\circ} \mathrm{C}$ for 1 hour after PLE was chosen. The highest concentrations of PFOS and PFOA were detected at $0.0671 \mathrm{ng} / \mathrm{g}$ and 21.0644 $\mathrm{ng} / \mathrm{g}$, respectively. Even though present concentrations are found at $\mathrm{ng} / \mathrm{g}$ levels, the daily use of sunscreen products is normally several grams. Therefore, a risk assessment of PFOS and PFOA contamination in sunscreen products is an important concern, and more attention needs to be paid to the long-term effects on human health.
\end{abstract}

Keywords: Centrifugation, LC-MS/MS, Personal care products, Perfluorinated compounds, Pressurized liquid extraction (PLE), Solid phase extraction (SPE)

\section{Introduction}

Perfluorinated compounds (PFCs) are fully fluorinated. Perfluorooctane sulfonate (PFOS) and Perfluorooctanoic acid (PFOA) are the most frequently detected PFCs [1]. PFOS has recently been included on the list of restricted-use compounds that appear in Annex B of the Stockholm Convention on Persistent Organic Pollutants, May 2009 [2]. The structure of PFOS and PFOA reflects a high electronegativity of fluorine atoms; the carbon-fluorine bond generates a strong dipole moment [3]. This characteristic is very stable at high temperatures, making it resistant to breakdown in the environment, bases, or oxidizers [4]. They have been widely used for a variety of applications such as in textiles, clothes, carpets, paper coatings, waterproofing agents, foam, and cosmetics [5]. PFOS and PFOA are released into the environment by industries

This is an Open Access article distributed under the terms of the Creative Commons Attribution Non-Commercial License (http://creativecommons. org/ licenses/by-nc/3.0/) which permits unrestricted non-commercial use, distribution, and reproduction in any medium, provided the original work is properly cited.

Copyright (C) 2015 Korean Society of Environmental Engineers and consumers, and these compounds are bio-accumulative, highly persistent, and toxic. There have been several reports of PFOS and PFOA being detected in wastewater, foodstuffs, animals, and blood. In the northern Bohai Sea in China, researchers investigated PFCs in water, soils, sediments, and biota collected from estuarine and coastal areas. The concentration of PFCs found in water (mean: $18.4 \mathrm{ng} / \mathrm{L}$ ) and biological samples (fish: $265 \mathrm{ng} / \mathrm{g} \mathrm{DW}$ ) were relatively high while the concentration of PFCs in soils and sediments was less [6]. In Japan concerning about the contamination of the PFOS and PFOA in surface water from the Yodo River, the concentrations measured were 0.4-123 ng/L and 4.2-2,600 ng/L, respectively [7]. In Italy, there have been reports of levels of PFOS at $0.76 \pm 1.27$ $\mathrm{ng} / \mathrm{g}$ and PFOA at $8.04 \mathrm{ng} / \mathrm{g}$ in breast milk, but only PFOS was found in food samples [8]. PFCs are also sometimes used in water-resistant sunscreen products. Sunscreen protects against ultraviolet
Received May 29, 2014 Accepted February 19, 2015

$\uparrow$ Corresponding author

Email: suwanna.boo@mahidol.ac.th

Tel: +66-2-889-2138 ext. 6390 Fax: +66-2-889-2138 ext. 6388 
rays and against sunburn from sunlight. The 'water-resistant' designation means that the sunscreen retains its stated SPF value after a certain time in water or while sweating. However, there are concerns that the ingredients of sunscreen can cause many adverse health effects. Following exposure to any chemical, the adverse health effects that may be encountered depend on several factors, e.g., the amount of exposure (dose), the way of exposure, the duration of exposure, and the form of the chemical. With regard to PFOS contamination in the European Union, the following point was added to Annex I of Directive 76/69/EEC [9]: that (certain chemicals/PFOS and PFOA) may not be placed on the market or used as a substance or constituent of preparations in a concentration equal to or higher than $0.005 \%$ by mass. Sunscreen is a semi-liquid, with high protein and organic content, which makes it difficult to analyze. One of the obstacles to identifying PFOS and PFOA contamination in semi-liquid phase products like sunscreen is too little information regarding the measurement method. In the group of solids such as food, sludge, and soil, the pressurized liquid extraction (PLE) technique has been used, followed by solid phase extraction (SPE) before analysis with liquid chromatography coupled with tandem mass spectrometry (LC-MS/MS) [10, 11]. PFOS and PFOA have been measured from food packaging, foods, and textiles by the PLE technique, followed by centrifugation and analysis by LC-MS/MS [12]. These two principal methods have advantages and disadvantages in terms of chemical contamination, processing time, detection level, and matrix effects in LC-MS/MS. Therefore, this research aims to develop a method by comparing SPE and centrifugation methods after PLE and to investigate concentrations of PFOS and PFOA in semi-liquid phase samples like waterproof sunscreen.

\section{Materials and Methods}

\subsection{Standards and Reagents}

The majority of analytical standards of perfluorooctane sulfonate (PFOS standard of 98\% purity) and perfluorooctanoic acid (PFOA standard of $>95 \%$ purity) used in this study were purchased from the Wako company (Japan). Pure ammonium acetate was ordered from the USA and used for preparing the mobile phase in the LC-MS/MS. In addition, Methanol high performance liquid chromatography (HPLC) grade used for extraction and Acetonitrile HPLC grade were supplied from Merck (Germany).

\subsection{Sunscreen Samples}

All samples were obtained randomly from domestic brands and international brands in stores in Thailand for this study. A total of 12 samples of different brands of sunscreen from many countries were made. In addition, all samples of sunscreen were chosen because they had water resistant, sweat resistant, waterproof, or non-stick properties. These samples were not previously used and were newly opened for analysis in this study.

\subsection{Sample Preparation by Pressurized Liquid Extraction Technique}

In this study, the amount of sunscreen sample used in each ex- traction was weighed on foil, about 4-5 g, and the sample was transferred into a $33 \mathrm{~mL}$ stainless steel cell. Stainless steel balls were used as filler to reduce the volume within the stainless steel cell.

The extraction of PFOS and PFOA from sunscreen samples was performed by Pressurized Liquid Extraction (PLE), using the Dionex ASE 200 accelerated solvent extraction system (ASE). Briefly, 4-5 g of each sample was weighed and prepared in duplicate. Then, each sample was transferred into a $33 \mathrm{~mL}$ stainless steel extraction cell. Cellulose filters were placed at the bottom of the extraction cell to avoid obstruction of the metal filters by solid particles. The cell was filled up with stainless steel balls and loaded into the ASE. In this work, HPLC grade methanol was used as a PLE solvent for extraction of all concentrations of PFOS and PFOA in the samples. The extraction technique was optimized and validated by the absolute recovery of PFOS and PFOA fortified control samples at two different levels (5 and $30 \mathrm{ng} / \mathrm{g}$ ). The 4-5 g samples were fortified by spiking $0.5 \mathrm{~mL}$ of PFOS and PFOA fortification solution at a concentration level of $10 \mu \mathrm{g} / \mathrm{L}$ and 60 $\mu \mathrm{g} / \mathrm{L}$. Three replicates of four samples were performed at each level. The PLE conditions were preheating for $0 \mathrm{~min}$, heating for $5 \mathrm{~min}$, and $30 \mathrm{~min}$ of static extraction time. 100\% flush volume and one extraction cycle at $80^{\circ} \mathrm{C}$ with 1,000 psi were set up. After extraction by PLE, each of the extracted samples dissolved in methanol was decanted into a $60 \mathrm{~mL}$ polypropylene bottle. Most samples were analyzed shortly after preparation. Otherwise, they were stored at $4^{\circ} \mathrm{C}$ and analyzed within a week.

\subsection{Preparation Method of Extracted Sample for Analysis by Solid Phase Extraction (SPE)}

After the PLE technique, the two alternative methods of treatment used before analysis by LC-MS/MS were SPE and centrifugation. The workflow of the SPE method focused on treating samples by SPE to concentrate and purify samples for analysis, and ENVI-Carb SPE Tubes ( $3 \mathrm{~mL}$ and $0.25 \mathrm{~g}$; Supelco, USA) were used to eliminate interference and remove organic matter [13] as shown in Fig. 1(a). After the PLE technique, $60 \mathrm{~mL}$ from the liquid phase was completely dried by water bath at $100^{\circ} \mathrm{C}$ to evaporate the methanol. Ultrapure water $(500 \mathrm{~mL})$ was added to the dried samples then they were loaded into the cartridge (Precep C-Agri C18; Wako, Japan) for SPE at a flow rate of 10 $\mathrm{mL} / \mathrm{min}$. After that, vacuum manifold was used to air dry the cartridge, and $4 \mathrm{~mL}$ of methanol followed by $2 \mathrm{~mL}$ of acetonitrile were added and then clean up by ENVI-Carb. Finally, the solution was purged by using high purity $\mathrm{N}_{2}$ gas until the sample in the vial completely dried. The dried samples were reconstituted by adding $0.5 \mathrm{~mL}$ of $40 \%$ acetonitrile and transferred into vials for analysis by LC-MS/MS. Fig. 1(b) shows the method wherein samples were completely dried and the volume adjusted to $5 \mathrm{~mL}$ for clean up by ENVI-Carb before the SPE step in order to prevent clogging from emulsion during SPE and compare the effectiveness of methods between cleaning up by ENVI-Carb before and after the SPE step. Then, the following processes were performed: cartridge drying, elution, $\mathrm{N}_{2}$ purging, reconstitution, and LC-MS/MS analysis. For each sample done, duplicate samples were also subjected to the same process. 


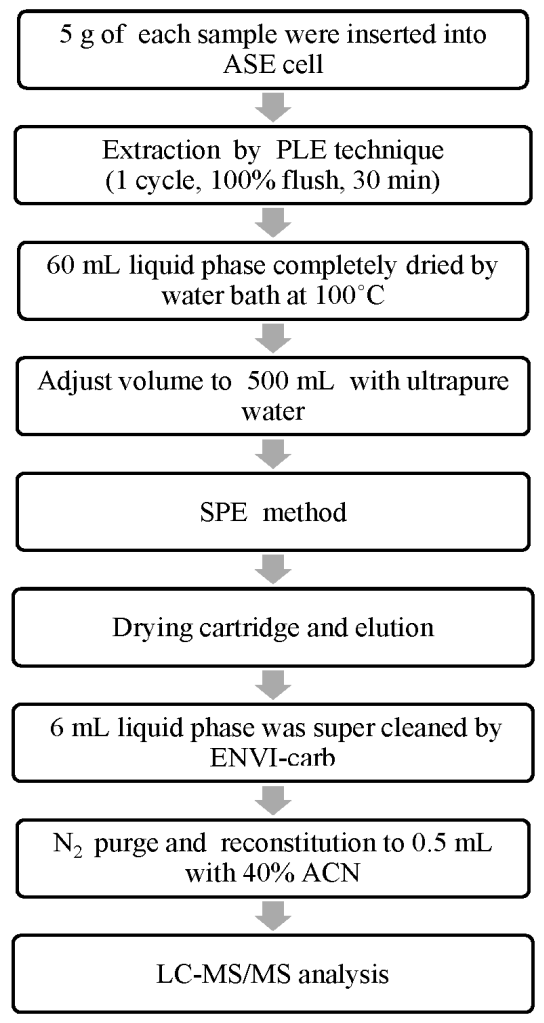

(a)

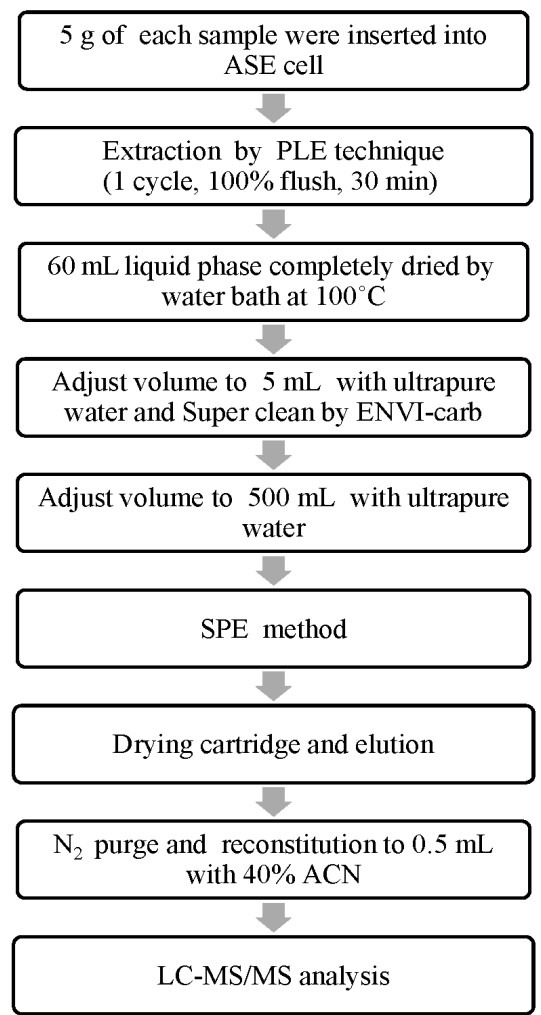

(b)

Fig. 1. Flowchart of preparation method by solid phase extraction (SPE)

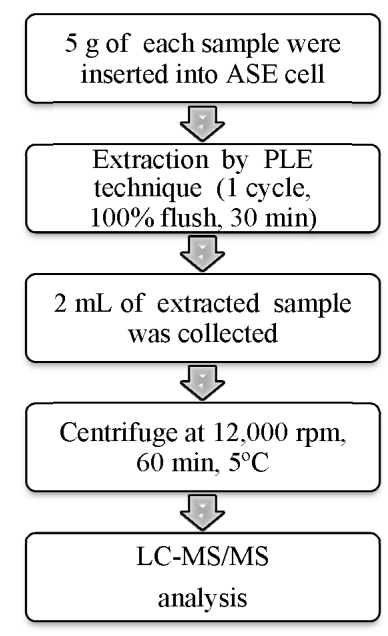

(a)

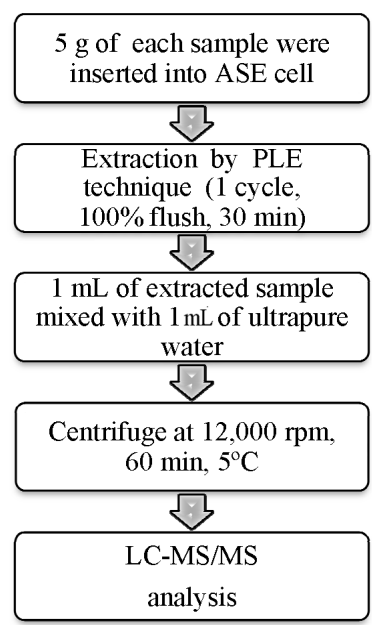

(b)

Fig. 2. Flowchart of preparation method by centrifugation (a) only $2 \mathrm{~mL}$ of extracted sample (b) addition of ultrapure water mixed with extracted sample.

\subsection{Preparation Method of Extracted Sample for Analysis by Centrifugation}

Two different centrifugation processes were used to separate lipids and remove potential matrix interference. After the PLE technique, $2 \mathrm{~mL}$ of extracted sample was placed into a $2 \mathrm{~mL}$ centrifuge tube and centrifuged at $12,000 \mathrm{rpm}$ and $5^{\circ} \mathrm{C}$ for 1 hour. After centrifugation, the upper, clear layer of the sample was transferred into a vial for analysis by LC-MS/MS as shown in Fig. 2(a). Fig. 2(b) shows $1 \mathrm{~mL}$ of extracted sample completely mixed with 1 $\mathrm{mL}$ of ultrapure water to help precipitation and perform emulsion phase separation [13]. The tubes were shaken to completely mix 
the contents homogenously and then centrifuged under the same conditions. After centrifugation, the upper, clear layer was transferred into a vial for analysis by LC-MS/MS.

\subsection{Instrumental Analysis}

Samples were analyzed by using Agilent 1200SL high performance liquid chromatography (HPLC, Agilent Technologies, Germany). The HPLC system was interfaced with an Agilent 6400 Triple Quadruple mass spectrometer (MS/MS, Agilent Technologies, USA). The analytical column used was Agilent Eclipse XDB-C $\mathrm{C}_{18}$, $4.6 \times 50 \mathrm{~mm}, 1.8 \mu \mathrm{m}$ and Plus $\mathrm{C}_{18}, 2.1 \times 100 \mathrm{~mm}, 1.8 \mu \mathrm{m}$. Analyte ions were analyzed by multiple reaction monitoring (MRM) mode. The mobile phase was performed using (A) $10 \mathrm{mM}$ ammonium acetate $\left(\mathrm{CH}_{3} \mathrm{COONH}_{4}\right)$ in ultrapure water and (B) acetronitrile (HPLC grade). The gradient conditions were as follows: the separation process started with an initial condition of $45 \%$ (B), ramping to $50 \%$ (B) at $5.0 \mathrm{~min}$, then increased to $60 \%$ (B) at $5.5 \mathrm{~min}$, held at $60 \%$ (B) for $4.5 \mathrm{~min}$, and rose to $90 \%$ (B) at $15 \mathrm{~min}$, at a flow rate of $0.25 \mathrm{~mL} / \mathrm{min}$. The column temperature was maintained at $40^{\circ} \mathrm{C}$ and the injection volume was $10 \mu \mathrm{L}$. PFOS and PFOA were

Table 1. Summary of Analytical Operation Conditions of HPLC-MS/MS

\begin{tabular}{ll}
\hline \multicolumn{1}{c}{ HPLC } & \multicolumn{1}{c}{ MS/MS } \\
\hline Instrument: & Instrument: \\
Agilent 1200 SL HPLC & Agilent 6400 triple quadrupole \\
& mass spectrometer
\end{tabular}

\section{Column:}

MS/MS operation: MRM

Agilent Eclipse $\mathrm{XDB}^{-\mathrm{C}_{18}}, 4.6 \times$

$50 \mathrm{~mm}, 1.8 \mu \mathrm{m}$ and Plus $\mathrm{C}_{18}$,

$2.1 \times 100 \mathrm{~mm}, 1.8 \mu \mathrm{m}$

Mobile Phase:

Source: ESI

A: $10 \mathrm{mM} \mathrm{CH}_{3} \mathrm{COONH}_{4} / \mathrm{H}_{2} \mathrm{O}$

B: $\mathrm{CH}_{3} \mathrm{CN}$

Flow: $0.25 \mathrm{~mL} / \mathrm{min}$

Gas flow: $10 \mathrm{~L} / \mathrm{min}$

Injection volume: $10 \mu \mathrm{L}$

Capillary voltage: $3,500 \mathrm{~V}$

Column temp: $40^{\circ} \mathrm{C}$

Gas temp: $300^{\circ} \mathrm{C}$

HPLC: high performance liquid chromatography, MS/MS: mass spectrometer, MRM: multiple reaction mode, ESI: electrospray ionization. detected using electrospray ionization (ESI). The operating conditions for ESI were as follows: gas flow was $10 \mathrm{~L} / \mathrm{min}$ with a capillary voltage of $3,500 \mathrm{~V}$ and gas temperature at $300^{\circ} \mathrm{C}$. The operation conditions are shown in Table 1. The calibration curve consisted of five concentration levels that ranged from $0.1-10 \mu \mathrm{g} / \mathrm{L}$. The total running time was $34 \mathrm{~min}$ for each sample, and all samples were stored in a refrigerator in polypropylene vials at $4^{\circ} \mathrm{C}$.

\section{Results and Discussion}

\subsection{Comparing the Results of Samples by Physical Observation}

Firstly, the preparation process of samples using SPE and centrifugation methods was physically observed. In the case of SPE method after clean up by ENVI-Carb, the sample was not passed through the ENVI-Carb because the emulsion was very viscous and finally could not be analyzed by LC-MS/MS. For the physical appearance of samples from the SPE method before cleaning by ENVI-Carb, the emulsion was observed as shown in Fig. 3(a), which was a significant problem encountered using this method. For the centrifugation method, low temperature $\left(5^{\circ} \mathrm{C}\right)$ and addition of water to the $1 \mathrm{~mL}$ sample can minimize the formation of an emulsion [13]. Only $2 \mathrm{~mL}$ of extracted sample was centrifuged for one hour at low temperature, which decreases the viscosity. For both methods of centrifugation, clear and bright samples were produced before analysis, as shown in Fig. 3(b). Because of the density difference between the emulsion and liquid phase of sample, centrifugal force separates the sample into the emulsion and liquid phase [14].

\subsection{Multiple Factors Considered for Method Selection}

This section compares the results of concentrations using SPE and centrifugation. Table 2 shows that the SPE method is an appropriate method for samples with low concentrations because SPE used the entire extracted sample (60 mL). Centrifugation could also detect PFOA in both samples, but PFOS contamination in sample 2 was not detected. Based on the results from the two different methods (SPE and centrifugation), both methods were able to detect the PFOS and PFOA concentrations at the ng/g levels. Even so, SPE could measure at lower levels than centrifugation, but there was more damage to the LC-MS/MS as more
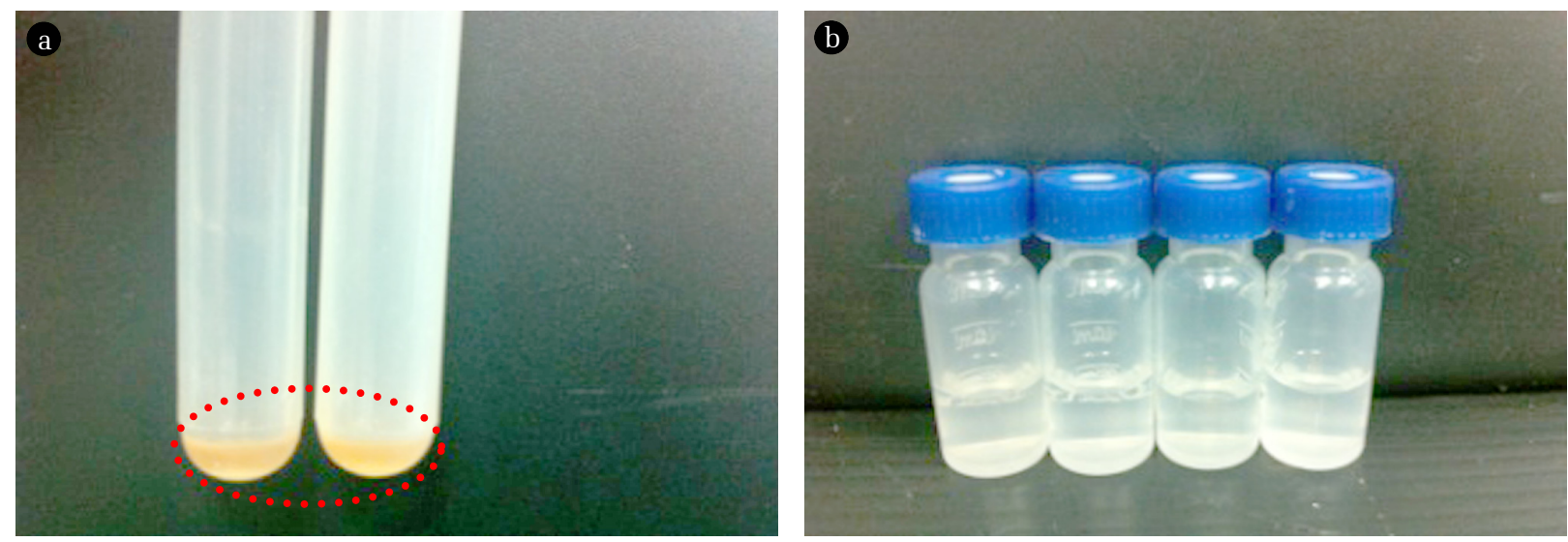

Fig. 3. Extracted samples for LC-MS/MS analysis using (a) solid phase extraction (SPE) and (b) centrifugation. 
emulsion and organic compounds remained and clogged the analytical column. Some of these spread into other parts of the detector, disturbing the operation of the detector.

Table 3 represents the correlation parameters between SPE and centrifugation methods. The parameters considered for optimization were operation time, chemical consumption in the process, and physical properties. The SPE method was performed and took about 8 hours, which was considered a long period compared with the centrifugation method ( 2 hours). A higher volume of chemicals, both methanol and acetonitrile, were used in the SPE method. Due to its physical properties, the emulsion remained stable throughout the whole period of study by SPE. Clear samples were observed from both centrifugation methods. The advantages of the centrifugation method were the shorter operation time and less volume of chemical consumption. Moreover, the extracted samples were clear and had less effect on LC-MS/MS.

Table 4 shows that PFOA and PFOS were detected in most of the waterproof sunscreen samples. Centrifugation with only $2 \mathrm{~mL}$ of extracted sample could detect PFOA in all samples, but PFOS contamination in one sample was not detected. The highest concentrations of PFOA and PFOS were found at the levels of $21.0644 \mathrm{ng} / \mathrm{g}$ and $0.0671 \mathrm{ng} / \mathrm{g}$, respectively. In the case of the centrifugation method using an extracted sample mixed with ultrapure water, the highest concentration of PFOA was found at

Table 2. Comparing Concentrations of PFOS and PFOA in Waterproof Sunscreen Samples by SPE and Centrifugation Methods

\begin{tabular}{|c|c|c|c|c|c|c|}
\hline \multirow{3}{*}{ Sunscreen samples } & \multirow{2}{*}{\multicolumn{2}{|c|}{ SPE method (ng/g) }} & \multicolumn{4}{|c|}{ Centrifugation method (ng/g) } \\
\hline & & & \multicolumn{2}{|c|}{$2 \mathrm{~mL}$ of extracted sample } & \multicolumn{2}{|c|}{ Extracted sample + ultrapure water } \\
\hline & PFOA & PFOS & PFOA & PFOS & PFOA & PFOS \\
\hline Sample 1 & 0.1564 & 0.0078 & 0.5865 & 0.0381 & 0.4627 & 0.0763 \\
\hline Sample 2 & 0.0396 & 0.0054 & 0.0485 & ND & 0.0281 & $\mathrm{ND}$ \\
\hline
\end{tabular}

SPE: solid phase extraction, PFOA: perfluorooctanoic acid, PFOS: perfluorooctane sulfonate, ND: not detected.

Table 3. Factors Considered for Optimization

\begin{tabular}{lccc}
\multicolumn{1}{r}{ Parameter } & SPE method & \multicolumn{2}{c}{ Centrifugation } \\
\cline { 3 - 4 } Time (h) & 8 & 2 mL of extracted sample & Extracted sample + ultrapure water \\
Chemical & & 2 & 2 \\
- MeOH (mL) & 64 & 60 & 60 \\
- ACN (mL) & 2 & 0 & 0 \\
Physical property & emulsion & clear & clear \\
Concentration & ng/g & ng/g & ng/g \\
\hline
\end{tabular}

Table 4. Concentrations of PFOS and PFOA in Waterproof Sunscreen Samples by Centrifugation Methods

\begin{tabular}{|c|c|c|c|c|c|c|}
\hline \multirow{3}{*}{$\begin{array}{l}\text { Water proof } \\
\text { sunscreens }\end{array}$} & \multirow{3}{*}{ SPF } & \multirow{3}{*}{ Country of origin } & \multicolumn{4}{|c|}{ Concentration (ng/g) } \\
\hline & & & \multicolumn{2}{|c|}{$2 \mathrm{~mL}$ of extracted sample } & \multicolumn{2}{|c|}{ Extracted sample + ultrapure water } \\
\hline & & & PFOA & PFOS & PFOA & PFOS \\
\hline Sample 1 & 50 & USA & 0.5865 & 0.0381 & 0.4627 & 0.0763 \\
\hline Sample 2 & 30 & Thailand & 0.0485 & ND & 0.0281 & $\mathrm{ND}$ \\
\hline Sample 3 & 130 & China & 0.0107 & 0.0274 & $\mathrm{ND}$ & ND \\
\hline Sample 4 & 36 & Philippines & 0.8526 & 0.0353 & 1.2098 & 0.1072 \\
\hline Sample 5 & 50 & Japan & 0.0318 & 0.0671 & 0.0268 & $\mathrm{ND}$ \\
\hline Sample 6 & 40 & Thailand & 21.0644 & 0.0233 & 142.8350 & $\mathrm{ND}$ \\
\hline Sample 7 & 30 & Thailand & 0.7681 & 0.0584 & 1.4193 & 0.1169 \\
\hline Sample 8 & 50 & Thailand & 1.1117 & 0.0629 & 1.4202 & $\mathrm{ND}$ \\
\hline Sample 9 & 50 & Thailand & 0.7017 & 0.0402 & 0.9600 & 0.1218 \\
\hline Sample 10 & 60 & Thailand & 2.0145 & 0.0229 & 2.8609 & 0.0892 \\
\hline Sample 11 & 50 & USA & 0.6365 & 0.0565 & 1.0312 & 0.0617 \\
\hline Sample 12 & 50 & Australia & 0.9685 & 0.0390 & 1.7513 & 0.0457 \\
\hline \multicolumn{3}{|c|}{ Max (ng/g) } & 21.0644 & 0.0671 & 142.8350 & 0.1218 \\
\hline \multicolumn{3}{|c|}{ Min (ng/g) } & 0.0107 & ND & $\mathrm{ND}$ & $\mathrm{ND}$ \\
\hline \multicolumn{3}{|c|}{ Recovery percentage } & 101.75 & 64.32 & 117.49 & 67.50 \\
\hline \multicolumn{3}{|c|}{$\%$ RSD } & 9.92 & 15.01 & 5.21 & 19.84 \\
\hline
\end{tabular}

PFOA: perfluorooctanoic acid, PFOS: perfluorooctane sulfonate, ND: not detected. 
$142.8350 \mathrm{ng} / \mathrm{g}$ and PFOS at $0.1218 \mathrm{ng} / \mathrm{g}$. The centrifugation method with the addition of ultrapure water mixed with the extracted sample could not detect PFOS contamination in 5 samples. The average percent recovery for the centrifugation method with 2 $\mathrm{ml}$ extracted samples were $101.75 \%$ and $64.32 \%$ for PFOA and PFOS, respectively, while extracted samples mixed with ultrapure water provided average recovery at $117.49 \%$ for PFOA and $67.5 \%$ for PFOS. Compared to other reports, the highest PFOA levels detected in sunscreen in this study were lower than the highest concentration of PFOA in Japanese research [15] for the samples listed as polyfluoroalkyl phosphate esters (PAPs) and other fluorinated compounds but were comparable to the samples not listed as fluorinated compounds.

\section{Conclusions}

The SPE and centrifugation methods were tested and compared for detecting the presence of PFOA and PFOS in waterproof sunscreen samples. Four primary factors were considered in order to select the optimal method. Firstly, regarding the physical properties, the samples resulting from centrifugation were better and clearer. Secondly, considering concentration levels, both SPE and centrifugation methods were able to detect concentrations at $\mathrm{ng} / \mathrm{g}$ levels, but the centrifugation method was selected because it was safer with regard to the LC-MS/MS machine. Thirdly, after considering multiple factors, the two different centrifugation methods provided optimal properties, which were shorter operation times, lower chemical consumption, and better physical properties. Lastly, the concentration level was in the same range, but the percent recovery from centrifugation using $2 \mathrm{~mL}$ of extracted sample was closer to 100 percent than the other. Therefore, the centrifugation method using only $2 \mathrm{~mL}$ of extracted sample with the conditions of $12,000 \mathrm{rpm}$ and $5^{\circ} \mathrm{C}$ for 1 hour was the method selected. The highest concentrations of PFOA and PFOS were found at the level of $21.0644 \mathrm{ng} / \mathrm{g}$ and $0.0671 \mathrm{ng} / \mathrm{g}$, respectively. This is the first report on the extraction method for the analysis of PFOS and PFOA in waterproof sunscreen samples in Thailand. The safety concerns regarding the effects of PFOS and PFOA on human health should be studied for any adverse health effects, and a risk assessment of contamination in personal care products including waterproof sunscreens should be done.

\section{References}

1. Jogsten IE, Perelló G, Llebaria X, et al. Exposure to perfluorinated compounds in Catalonia, Spain, through consumption of various raw and cooked foodstuffs, including packaged food. Food Chem. Toxicol. 2009;47:1577-1583.

2. Liu X, Jin Y, Liu W, Wang F, Hao S. Possible mechanism of perfluorooctane sulfonate and perfluorooctanoate on the release of calcium ion from calcium stores in primary cultures of rat hippocampal neurons. Toxicol. In Vitro 2011;25: 1294-1301.

3. Loccisano AE, Campbell JL, Andersen ME, Clewell HJ. Evaluation and prediction of pharmacokinetics of PFOA and PFOS in the monkey and human using a PBPK model. Regul. Toxicol. Pharmacol. 2011;59:157-175.

4. Florentin A, Deblonde $\mathrm{T}$, Diguio $\mathrm{N}$, Hautemaniere A, Hartemann P. Impacts of two perfluorinated compounds (PFOS and PFOA) on human hepatoma cells: Cytotoxicity but no genotoxicity? Inter. J. Hyg. Environ. Health. 2011;214:493-499.

5. Jin YH, Liu W, Sato I, Nakayama SF, Sasaki K, Saito N, Tsuda S. PFOS and PFOA in environmental and tap water in China. Chemosphere 2009;77:605-611.

6. Wang T, Khim JS, Chen C, et al. Perfluorinated compounds in surface waters from Northern China: Comparison to level of industrialization. Environ. Inter. 2012;42:37-46.

7. Lein NPH, Fujii S, Tanaka S, Nozoe M, Tanaka H. Contamination of perfluorooctane sulfonate (PFOS) and perfluorooctanoate (PFOA) in surface water of the Yodo River basin (Japan). Desalination 2008;226:338-347.

8. Guerranti C, Perra G, Corsolini S, Focardi SE. Pilot study on levels of perfluorooctane sulfonic acid (PFOS) and perfluorooctanoic acid (PFOA) in selected foodstuffs and human milk from Italy. Food Chem. 2013;140:197-203.

9. Directive E. Directive 2006/122/ECOF. the European Parliament and of the Council of 12 December 2006. Official J. Eur. Union. L 372 .

10. Zafeiraki E, Costopoulou D, Vassiliadou I, Bakeas E, Leondiadis L. Determination of perfluorinated compounds (PFCs) in various foodstuff packaging materials used in the Greek market. Chemosphere 2014;94:169-176.

11. Llorca M, Farré M, Picó Y, Barceló D. Development and validation of a pressurized liquid extraction liquid chromatography -tandem mass spectrometry method for perfluorinated compounds determination in fish. J. Chromatogra. A 2009;1216: 7195-7204.

12. Poothong S, Boontanon SK, Boontanon N. Determination of perfluorooctane sulfonate and perfluorooctanoic acid in food packaging using liquid chromatography coupled with tandem mass spectrometry. J. Hazard. Mater. 2012;205-206:139-143.

13. Ballesteros-Gómez A, Rubio S, van Leeuwen S. Tetrahydrofuran -water extraction, in-line clean-up and selective liquid chromatography/tandem mass spectrometry for the quantitation of perfluorinated compounds in food at the low picogram per gram level. J. Chromatogr. A 2010;1217:5913-5921.

14. Ropers MH, Durand S, Veyrand B, et al. Contamination of food by fluorinated surfactants - Distribution in emulsions and impact on the interfacial protein behaviour. Food Hydrocoll. 2009;23:1149-1155.

15. Fujii Y, Harada KH, Koizumi A. Occurrence of perfluorinated carboxylic acids (PFCAs) in personal care products and compounding agents. Chemosphere 2013;93:538-544. 